\title{
Validación de una escala para medir la competencia digital en estudiantes de posgrado
}

\author{
Martha O. Ramírez-Armenta1, Ramona I. García-López ${ }^{1 *}$ y Rubén Edel-Navarro ${ }^{2}$ \\ (1) Depto. de Educación, Instituto Tecnológico de Sonora, 5 de Febrero 818 sur, CP 85000 , Sonora, México \\ (Correo-e: martha.ramirez@potros.itson.edu.mx; igarcia@itson.edu.mx)) \\ (2). Facultad de Pedagogía. Universidad Veracruzana. Calz. Juan Pablo II, 1193, Costa Verde, CP 94294 Boca del Río, \\ Ver, México (Correo-e: redel@uv.mx)
}

*Autor a quien debe ser dirigida la correspondencia

Recibido Nov. 30, 2020; Aceptado Ene. 25, 2021; Versión final Mar. 2, 2021, Publicado Jun. 2021

\begin{abstract}
Resumen
El objetivo de este estudio es desarrollar una escala válida y confiable para medir la competencia digital, aplicada al proceso investigativo, en los estudiantes de posgrado orientados a la investigación. Se utiliza un método mixto de dos fases: en la primera (cualitativa) participaron 51 investigadores reconocidos por su trayectoria a nivel nacional; se les aplicó una entrevista para determinar las dimensiones que componen a la competencia investigativa. En la segunda fase (cuantitativa), se desarrollaron las escalas de cada una de las dimensiones identificadas en la fase 1, pero para los fines de esta investigación, solo se presenta la escala para medir la competencia digital. Participaron 323 estudiantes de diferentes programas educativos. Los resultados indican que las categorías de la competencia digital son la competencia informacional y el uso de herramientas tecnológicas. Se concluye que la escala diseñada cumple con los criterios de validez de constructo y confiabilidad pertinentes.
\end{abstract}

Palabras clave: competencia digital; posgrado; educación superior; confiabilidad en medición

\section{Validation of a scale to measure digital competence in graduate students}

\begin{abstract}
The objective of the present study is to develop a valid and reliable scale to measure digital competence, applied to the research process, in research-oriented graduate students. A two-phase mixed method is used. In the first phase (qualitative), 51 researchers, known by their trajectory at national level, are interviewed to determine dimensions involved in research competence. In the second phase (quantitative), scales are developed for each of the dimensions identified in phase one, but for the purposes of this study, only the scale for measuring digital competence is presented. A total of 323 students from different educational programs are surveyed. The results show that the categories of digital competence are information competence and technological tools use. It is concluded that the scale developed here meets both construct validity and reliability criteria.
\end{abstract}




\section{INTRODUCCIÓN}

La educación superior es clave para cualquier país, pues en ella se desarrollan las competencias generales y específicas, así como los conocimientos técnicos, profesionales y disciplinares necesarios para insertarse en el campo laboral. En el caso específico de México, este nivel educativo enfrenta retos importantes en cuanto a calidad se refiere; existen diversas instancias gubernamentales y sociales que tratan de regular su funcionamiento y proponer mecanismos que les permitan evaluar su desarrollo y lograr los estándares establecidos para su certificación y acreditación (López, 2020).

En México, existen algunas iniciativas para mejorar la educación superior, tales como: el Programa de Fortalecimiento a la Excelencia Educativa (Profexce); el Consejo Nacional de Ciencia y Tecnología (Conacyt); Programas de incubadoras de empresas por parte de la Secretaría de Economía; la iniciativa de la Cámara de diputados Fortalecimiento a la Educación Superior y la Capacitación para Impulsar el Desarrollo y la Competitividad (CEFESCDC), entre otros. Sin embargo, todas ellas se ejecutan de forma aislada y no trabajan bajo un marco cohesivo. Además, no se han implementado acciones de evaluación y monitoreo del rendimiento y eficacia de tales estrategias, con el fin de mejorar sus resultados e impactar en la calidad educativa (OCDE, 2019).

Con el objeto de asegurar la calidad en dicho nivel educativo, se han llevado a cabo diferentes estrategias por parte del Gobierno para medir su eficiencia. Un ejemplo de ello es el Programa Nacional de Educación Superior (Pronaes) que surge en $1984 \mathrm{con}$ el fin de apoyar proyectos que se consideraban de calidad. Posteriormente, se implementa el Programa de Fortalecimiento del Posgrado Nacional (PFPN), cuyo objetivo era mejorar la calidad mediante la asignación de apoyos financieros complementarios En 1986 se desarrolla el Programa Integral de Desarrollo de la Educación Superior (Proides) donde se estandarizaron criterios de evaluación para medir la calidad y el uso eficiente de los recursos financieros (Mireles, 2008).

En la educación superior se desarrollan estudios de técnico superior, licenciatura y posgrado. En el primero se forman profesionales capacitados para el trabajo dentro de un área específica; son de carácter terminal y no alcanzan el nivel de licenciatura. La licenciatura se cursa en cuatro años o más y se orienta a la formación de capital humano para el ámbito profesional en diversas áreas del conocimiento. El posgrado abarca los estudios de especialidad, maestría y doctorado; se encarga de formar investigadores y profesionistas con alto grado de especialización. En el caso del nivel de posgrado, este ha ido evolucionando; en un primer momento se dio impulso al incremento en la matrícula; lo que generó un deterioro en el financiamiento otorgado debido a las pocas regulaciones. Después, surgió el interés por atender la calidad y se establece la evaluación como principio para lograrla; de esta forma, los recursos se compiten entre las instituciones a partir de los resultados obtenidos.

Actualmente en México, se le ha dado un impulso al desarrollo y consolidación del posgrado. Por ello, surge el Consejo Mexicano de Estudios de Posgrado (Comepo) para fortalecer y aumentar la cooperación académica entre las instituciones de educación superior (IES); así como desarrollar un proyecto donde se vinculen las necesidades del sector con los programas educativos del nivel y la calidad de los posgrados que se ofertan. En cuanto a educación se refiere, la universidad cumple uno de los papeles fundamentales en la creación y transmisión de conocimiento, ya que es en ella donde los futuros profesionistas adquirirán los saberes y habilidades que les servirán para desarrollarse como seres productivos dentro de una disciplina (Li, y Curdt-Christiansen, 2020). Es por esto que las IES deben preocuparse por formar a sus estudiantes integralmente; pero sobre todo de formarlos permanentemente proporcionándoles herramientas que les sirvan para seguir aprendiendo y actualizándose. Además, las exigencias hacia los egresados universitarios son cada vez más altas, por lo que es necesario desarrollar en ellos las competencias que sean compatibles con el mercado laboral. En este sentido, la formación que se da específicamente en el posgrado corresponde a un proceso educativo que tiene como fin lograr que los egresados sean capaces de construir su propio aprendizaje y contribuir al conocimiento científico. Es decir, se trata de proveer a los discentes con una formación avanzada, la cual puede ser con fines de especialización profesional y de investigación (Traiwichitkhun y Wongwanich, 2014).

A diferencia de otros grados académicos, un investigador se forma mediante la propia reflexión, se deben de considerar sus habilidades para desarrollarse y decidir sobre el propio rumbo y evolución, tiene capacidad de acción en el trabajo mismo; es decir, es partícipe de su propio proceso de formación y de la dirección que este debe tomar (Guerrero, et al., 2020). Un programa de posgrado con orientación hacia la investigación tiene como fin desarrollar investigadores competentes; es decir, proporcionar a los estudiantes conocimientos y habilidades para llevar a cabo procesos de investigación y difundirla en un campo particular de la ciencia (Ismail y Mohd, 2012). Para este trabajo, se considera que la formación de los estudiantes de posgrado debe incluir habilidades específicas que fortalezcan la competencia para la investigación independientemente del área de la ciencia en la que se estén formando. 
Por ello, se entiende por competencias de investigación a la capacidad de manejar y utilizar el conocimiento científico, la identificación de cuestionamientos y obtención de conclusiones fundamentadas en pruebas que permiten la comprensión del mundo natural o los cambios generados por la actividad humana con el fin de orientar la toma de decisiones (Olehnovica, et al., 2014). Al revisar la literatura existente del tema se pueden encontrar diversos trabajos que describen las competencias que todo investigador debe dominar para poder desempeñarse en la profesión. La mayoría coincide con los elementos que componen tal competencia y las diferencias se encuentran en cómo se denomina la habilidad y los límites entre una y otra (Asia-Pacific Economic Cooperation, 2010).

Particularmente, en esta investigación solo se aborda la competencia digital por considerarla una competencia transversal que todo investigador en formación debe desarrollar, independientemente del área disciplinar en el que se encuentre. Al respecto, cabe mencionar que la Organización para la Cooperación y el Desarrollo Económico (OCDE), generó un reporte donde presentó datos estadísticos sobre los estudiantes mexicanos y otros 31 países, sobre el uso de las computadoras y su aprendizaje de temas específicos (lectura, habilidades digitales y enseñanza de matemáticas). En dicho documento se señala que la comunidad educativa debe integrar la tecnología en los procesos de enseñanza-aprendizaje, de tal manera que apoye las metodologías académicas (OCDE, 2019). Por su parte, López (2020) menciona que las competencias digitales tendrán un impacto abrupto en todos los ámbitos de la sociedad en los siguientes años. Por ello, todas las instituciones educativas deberán poner especial énfasis en su desarrollo a través del currículum de todos sus programas educativos. Al respecto, diversos investigadores y organizaciones, han propuesto diferentes clasificaciones para facilitar su estudio, así como una variedad de instrumentos que permitan su medición.

\section{OTROS ANTECEDENTES}

Hay una serie de antecedentes adicionales que es necesario detallar para documentar en mejor forma este trabajo. Estos se describen en dos subsecciones: i) fundamentación teórica; y ii) planteamiento del problema.

\section{Fundamentación teórica}

Distintas organizaciones e instituciones a nivel internacional han evidenciado la importancia de la competencia digital y han determinado diversos estándares para alumnos y docentes. Un ejemplo de ello es la International Society for Technology in Education (ISTE), organismo que genera normativas sobre los dominios que diversos actores educativos deben poseer en cuanto a competencia digital, que les sirvan para ser eficientes en la vida y el trabajo. El marco que este organismo declara para los estudiantes se conforma de dimensiones como: creatividad e innovación, comunicación y colaboración, investigación y manejo de información, pensamiento crítico, ciudadanía digital y operación de la tecnología (ISTE, 2015). Por otro lado, la Unesco (2013) considera que la tecnología permitirá que profesores y alumnos alcancen el éxito en el propio aprendizaje, en el trabajo y por añadidura en la vida cotidiana. $Y$ es por ello que pondera a la competencia digital tan alto pues es por medio de ella que se eleva la productividad y se genera la innovación. Para esta institución es necesario que los estudiantes tengan experiencias que incluyan el uso de tecnología como medio para aprender, utilizar distintos recursos tecnológicos, enriquecer sus habilidades para el manejo de las tecnologías de la información y comunicación (TIC), entre otros.

Respecto a las dimensiones que conforman la competencia digital Blau, et al. (2020) señalan que para evaluar la competencia en el tratamiento de la información y competencia digital es necesario considerar cinco dimensiones: la informacional, del aprendizaje, la comunicativa, la cultura digital y la tecnológica. Van, et al. (2020), mencionan que la competencia digital comprende cuatro dimensiones: 1) utilizar internet para obtener información, 2) clasificar y organizar la información utilizando la computadora, 3) construir y comunicar conocimiento, utilizando la computadora y 4) reconocer programas básicos con los que ha trabajado anteriormente y para qué le pueden servir. La Comisión Europea generó un marco sobre competencias digitales en los ciudadanos, donde propone 5 dimensiones para abordarla: alfabetización informacional y manejo de datos; comunicación y colaboración; creación de contenido digital; seguridad y resolución de problemas (Carretero, et al., 2017). Por su parte el Joint Informatios Systems Committee (2018) presenta 6 elementos definidos para el desarrollo de capacidades digitales donde incluye dimensiones como: competencia en TIC, información, datos y alfabetización mediática, creación digital, resolución de problemas e innovación, comunicación, colaboración y participación, aprendizaje y desarrollo digital e identidad digital y bienestar.

Por otra parte, Olehnovica et al. (2013), comentan que está constituida por cinco dimensiones: a) aprendizaje, contempla conocimiento y adquisición; b) informacional, es la adquisición, evaluación y uso de la información en ambientes digitales; c) comunicativa, consiste en la comunicación interpersonal y social; d) cultura digital, concierne a la práxis social y cultural del conocimiento en ambientes digitales; y e) tecnológica, tiene referencia al conocimiento y dominio tecnológico en entornos virtuales. Coincidentemente, Basantes, et al. (2020) 
consideraron que dicha competencia se conforma por los constructos: alfabetización informacional, comunicación y colaboración, creación de contenido digital, seguridad y resolución de problemas. Por último, la Coordinación de Tecnologías para la Educación de la Universidad Nacional Autónoma de México propone la matriz de habilidades digitales. Misma que se conforma por 8 dimensiones: acceso a la información; comunicación y colaboración en línea, seguridad de la información, procesamiento y administración de la información, manejo de medios, equipos de cómputo y dispositivos móviles, ambientes virtuales de aprendizaje, recursos y herramientas tecnológicas de apoyo a la enseñanza (DGTIC, 2014).

Al revisar la literatura se encontraron escalas que miden tal competencia desde diferentes puntos de vista y en diferentes niveles educativos. Tal es el caso del estudio de Beltrán, et al. (2017), quienes validaron una escala para medir la competencia informacional en docentes de educación básica. Pinto, et al. (2012) trabajaron con la misma competencia pero en estudiantes de posgrado con una escala de percepción; para ello, diseñaron el instrumento ALFINVES incluyendo indicadores relacionados al saber buscar, encontrar, evaluar y utilizar información con el fin de resolver necesidades. Por otra parte, Bolatovna, et al. (2016), analizaron la competencia en TIC de alumnos de maestría. Se identificaron las actividades que los estudiantes realizan con el apoyo de tecnología para desarrollar acciones de la investigación científica.

Como puede observarse, existen diferentes propuestas que dicen medir la competencia digital de distintas poblaciones, niveles de estudio y con diversos dominios; pero en general, las dimensiones coinciden entre unas y otras; las principales diferencias radican en la concepción de los constructos de lo que se considera constituye la tecnología como competencia humana. Sin embargo, para este estudio, el interés se centraba en conocer cuáles son las competencias digitales que requiere un investigador para llevar a cabo las actividades propias de su profesión; todo esto desde el punto de vista de un experto, en este caso el investigador consolidado. Si bien algunas dimensiones pueden coincidir, la diferencia se presenta en esas actividades específicas que solo realiza quien se desempeña como generador de conocimiento y esos indicadores eran los que interesaban conocer.

Por ello, se revisaron distintas propuestas que miden la competencia digital pero la mayoría se enfocan en los niveles previos a los estudios de posgrado y los que se centran en este nivel se realizaban en una determinada área de conocimiento.Cabe aclarar que lo que se reporta en este documento es solo una parte (la que corresponde a la competencia digital) de un estudio mayor que se interesaba en identificar todas las habilidades que conforman la competencia de investigación; entendida esta como todas las capacidades que componen el quehacer del investigador y que independientemente de la disciplina en la que se desarrolle la actividad científica, todos deben dominar por igual. Bajo esa premisa y con el fin de identificar las habilidades necesarias para el desarrollo de dicha competencia, se buscaron instrumentos que evaluaran y definieran sus elementos. Los instrumentos recopilados consideran varias de las habilidades en común y difieren en otras. Entre las que tienen una mayor mención son las metodológicas, comportamiento ético y procesos de revisión, difusión y escritura de textos científicos; en las que los autores difieren son profesionalismo, uso de tecnología (competencia digital) y liderazgo.

\section{Planteamiento del problema}

Desde una concepción sistémica de la educación es necesario estar en constante razonamiento y abordar las problemáticas desde la educación básica hasta la superior; pues ambas son elementales para mejorar el sistema educativo y de esta forma repercutir en el desarrollo de la nación. En materia de desarrollo científico toda aportación es importante, desde analizar las políticas, los aspectos presupuestales, la apertura y el intercambio con el resto del mundo, el desarrollo tecnológico, etc., pero también es necesario atender los procesos mediante los cuales se forman las personas que desempeñarán las actividades relacionadas con la ciencia.

La evaluación de planes de estudio es una de las actividades con mayor relevancia dentro del contexto académico. En todos los niveles educativos, se monitorean las reformas implementadas, las nuevas estrategias y las innovaciones están a la orden del día, siempre atendiendo a las necesidades del contexto. Mediante este proceso se responde una diversidad de cuestionamientos que permiten reconocer las áreas de mejora, lo que funciona, el adecuado aprovechamiento de los recursos, los esfuerzos institucionales y las competencias de los actores (Goos y Salomons, 2017). Por lo anterior, las IES deben monitorear las demandas del contexto con el fin de ofertar la formación mínima necesaria al capital humano que ofrece a la sociedad del conocimiento. Este estudio parte de la necesidad de identificar cómo desde los estudios de posgrado se desarrollan las competencias requeridas para desempeñarse como investigadores, donde precisamente una de esas competencias es la digital; pues si bien esta ya ha sido estudiada desde distintos puntos de vista y con diversos actores educativos, se ha identificado que precisamenta a nivel posgrado su aplicación y desarrollo tiene distintas vertientes (Carrasco et al., 2015). 
En el caso de los estudios de posgrados, tales monitoreos en ocasiones quedan a disposición o son responsabilidad del núcleo de profesores que conforman la academia del programa. Dicha delegación puede deberse a que se considera que ellos son los expertos en el área y al tener este nivel de formación y profesionalización lo que ellos consideren es lo adecuado para la formación dentro de la disciplina.Si bien lo anterior es cierto, el reconocimiento a la experiencia de esos investigadores ya posicionados dentro de su área, corresponde al conocimiento profundo que tiene de la disciplina, el dominio del método científico y otros factores que son solo una parte de la formación que requieren los estudiantes de este nivel de estudio.

Por otro lado, también es determinante que los propios estudiantes y futuros investigadores sean conscientes de las competencias que pueden ofrecer al mercado laboral. $Y$ de igual forma, es indispensable que las empresas conozcan qué es lo que un investigador puede aportar a la industria; pues todavía se considera a este como experto, pero se carece de la comprensión de la amplia gama de habilidades que puede tener (Barthélémy y Matthieu, 2016). En razón de lo expuesto, es importante realizar estudios de este tipo para contar con resultados y evidencias que permitan, en primer lugar, proponer la formación en competencias genéricas en este nivel de estudios; concientizar a los maestros de las distintas disciplinas que hay contenidos ajenos a su ciencia que también deben de estar presentes en sus currículos y evidenciar cómo la realidad profesional exige el dominio de las competencias calificadas como genéricas del posgrado.

Por lo anterior, en la presente propuesta se pretende identificar cuáles son las habilidades que se relacionan con el uso de tecnología y que requiere un investigador con independencia del área de conocimiento para ejercer su profesión (es decir, se considera como una competencia transversal). Para ello, se desarrolla y valida una escala para medir la competencia digital, derivada de cómo se aplica en la formación de investigadores con el fin de contar con información pertinente que oriente la toma de decisiones en cuanto a la forma en que esta debe ser incorporada en el currículum de los programas educativos de posgrado.

\section{METODOLOGÍA}

Para llevar a cabo esta investigación se realizó un estudio mixto donde se recolectaron datos cualitativos mediante entrevistas (fase 1) e información cuantitativa a través de escalas (fase 2). A continuación se presenta las técnicas y métodos para la selección de los participantes, la recolección de los datos y el análisis de los mismos.

\section{Contexto de la investigación}

Este estudio se enmarca en un proyecto de mayor alcance, a partir de una investigación en donde uno de sus objetivos era identificar cuáles son las habilidades que componen la competencia para la investigación en los programas de posgrado orientados a la investigación en el estado de Sonora, México; esto desde la perspectiva de los investigadores consolidados y con base en esto, desarrollar las escalas que permitieran su medición en los estudiantes.

En Sonora, México se cuenta con 44 programas de posgrado en investigación, que son reconocidos por el Programa Nacional de Posgrados de Calidad (PNPC), avalados por el Consejo Nacional de Ciencia y Tecnología (Conacyt). Dichos programas son ofertados en las cuatro instituciones más reconocidas del estado: la Universidad de Sonora (Unison) con 30 programas, el Instituto Tecnológico de Sonora (Itson) con ocho; el Centro de Investigación en Alimentos y Desarrollo A. C. (CIAD) con cuatro y el Colegio de Sonora (Colson) con dos. Todos ellos, distribuidos en distintos campos disciplinarios, tales como ciencias de la salud, medio ambiente, recursos naturales, ingenierías y ciencias sociales. En México, existe el Sistema Nacional de Investigadores (SNI), que reconoce la trayectoria y desempeño de los investigadores mexicanos, quienes se someten a un proceso de evaluación riguroso para ocupar un nivel (Candidato, Nivel I, II y III) dentro de dicho sistema. El que los docentes-investigadores que forman parte de los Núcleos Académicos Básicos (NAB) de los programas educativos de posgrado, pertenezcan al SNI es un criterio de calidad importante para permanecer en el PNPC.

\section{Tipo de estudio}

Para la construcción de la escala se utilizó una metodología instrumental mediante un enfoque mixto secuencial, conformado por dos fases. En la primera se obtuvieron datos cualitativos por medio de entrevistas aplicadas a los investigadores miembros del SNI y que forman parte de las distintas instituciones educativas; lo anterior con el fin de determinar los indicadores que integrarían la escala. Con el objetivo de comprender el fenómeno de la competencia para la investigación desde la propia perspectiva del investigador, se analizaron los datos desde la fenomenología. En la fase dos, se construyeron las escalas a partir de los indicadores generados en la fase uno y lo identificado en la revisión de literatura; asimismo, se realizó la validación de dichas escalas. Cabe aclarar que en este estudio solo se presenta una de las escalas 
resultantes, la correspondiente a la competencia digital; $y$ no se reportan las otras competencias mencionadas por los investigadores, tales como: la metodológica, lingüística, ética y de gestión.

\section{Participantes}

En la fase 1, la muestra se conformó por dos investigadores de cada Núcleo Académico Básico de los programas de posgrado que se encuentran registrados con orientación a la investigación en el PNPC en el estado de Sonora. Los criterios para seleccionarlos fueron: que tuvieran el nombramiento del SNI y que uno fuera el investigador más novel y el otro con mayor experiencia de todo el NAB. En total, participaron 51 docentes: 32 hombres y 19 mujeres. A cada uno de los participantes se le asignó un código para identificarlos, conformado por el número de seriación según su posición dentro de la entrevista, la grafía $\mathrm{F}$ o $\mathrm{M}$ según el género y la grafía $\mathrm{E}$ o $\mathrm{N}$ si era considerado experto o novel según lo señalado por los coordinadores. Con respecto a la fase 2, participaron 323 estudiantes de los distintos programas de posgrado. La muestra fue probabilística por conveniencia y se conformó por 156 hombres y 167 mujeres; en edades entre 21 y 60 años, siendo 27 años la media de edad.

\section{Instrumentos}

El instrumento para la recolección de información en la fase 1 fue una entrevista semiestructurada; misma que se aplicó a los investigadores que forman parte de la academia que respalda a los programas de posgrado. Lo anterior, con la finalidad de conocer cuáles son todas esas competencias para la investigación que todo investigador debe dominar para desempeñarse adecuadamente en la profesión. La entrevista se basó en las siguientes preguntas: ¿Cuáles son las competencias que todo investigador debe tener para ejercer adecuadamente la profesión, independientemente de la disciplina en la que se desempeñe? De las competencias antes mencionadas, ¿qué orden de importancia le otorga a cada una? ¿Cuáles son los factores que influyen en el desarrollo de la competencia para la investigación en los estudiantes de posgrado? ¿Cuáles son los errores más comunes que presentan los estudiantes de posgrado al momento de realizar su tesis?

A partir de esta información se generaron los indicadores que se usaron en el diseño de las escalas que se desarrollaron posteriormente. En la fase 2 se diseñaron los instrumentos para medir las distintas competencias para la investigación que los docentes-investigadores consolidados determinaron como elementales para ejercer la profesión. Cabe señalar que para los fines de este estudio solo se presenta la escala para medir la competencia digital; esta estaba conformada por 14 ítems con 4 opciones de respuesta: mucho, bastante, poco y nada.

\section{Recolección de información}

Primeramente, se realizó un acercamiento con la coordinación de los programas educativos para solicitar la identificación de los integrantes de su NAB que cumplieran con el perfil solicitado. Una vez obtenida dicha información se les hizo llegar una invitación personal a los investigadores para participar en el proyecto. Al aceptar la invitación, se les solicitaba autorización para la grabación de la entrevista y la firma del consentimiento informado. De igual forma, en la fase 2 se solicitó autorización a los coordinadores de cada programa para tener acceso a los estudiantes de las instituciones donde se realizó la investigación; a quienes se les comunicaron los criterios de confidencialidad de los datos y se les solicitó su participación voluntaria y la firma del consentimiento informado. El instrumento se aplicó de manera impresa, pero también se tenía la versión digital, para los estudiantes que por alguna razón no se encontraran presentes en el momento de la aplicación.

\section{Análisis de datos}

En la fase cualitativa se utilizó el software Atlas. Ti; de este análisis se obtuvieron tanto los códigos como citas y categorías emergentes que conforman el constructo de la competencia digital. Para la fase cuantitativa se utilizó el software Statistical Package for the Social Sciences (SPSS) con el que se realizó la validación de la estructura interna del instrumento mediante la técnica multivariante del análisis factorial exploratorio (AFE). Después, se utilizó el software AMOS para confirmar el modelo de medida utilizando el análisis factorial confirmatorio (AFC) para obtener los criterios de bondad de ajuste propuestos por Hu y Bentler (1999).

\section{RESULTADOS}

Los resultados obtenidos a partir de la recolección de datos se presentan en las fases que componen el estudio. Primero, se muestran los resultados cualitativos del análisis de información derivada de las entrevistas realizadas a los investigadores. Después, se explican los resultados cuantitativos de la construcción del instrumento y los análisis para determinar las propiedades métricas del mismo. 


\section{Fase 1. Entrevista a investigadores}

La estructura de la escala que mide la competencia digital se conformó con la información obtenida de la entrevista realizada a los docentes investigadores de los posgrados. En el análisis de los datos se identificaron las citas que aportaban información importante y de ellas se establecieron códigos y categorías emergentes a las cuales se asociaron cada una de las citas. A la categoría relacionada con la competencia digital se asociaron dos códigos y 16 citas. Esta competencia consiste en la habilidad de saber cuándo se requiere información, cómo articular dicha necesidad informativa en términos propios y estandarizados por el contexto científico, para que mediante un vocabulario técnico se realice la búsqueda de información en la variedad de fuentes disponibles. Una vez encontrada, hay que realizar procesos de recuperación, interpretación, comprensión organización y evaluación de información (Meerah, et al., 2012).

El primer código que se generó se asoció a la alfabetización informacional; aquí los investigadores comentaron que es necesario tener habilidades para detectar una necesidad de información, buscar la información requerida; que tales búsquedas y revisiones sean sistemáticas, que se tengan criterios para seleccionar las fuentes y la información que sea verídica; así como estar en constante actualización, evaluar, procesar o saber qué hacer con todo lo recuperado. Algunas de las respuestas proporcionadas por los investigadores se presentan en la tabla 1.

Tabla 1. Respuestas de los investigadores asociadas a la alfabetización informacional

\begin{tabular}{|l|l|}
\hline Participante & \multicolumn{1}{c|}{ Respuesta } \\
\hline$(29, \mathrm{M}, \mathrm{E})$ & Deben saber dónde buscar la información en fuentes confiables. \\
\hline$(19, \mathrm{M}, \mathrm{N})$ & $\begin{array}{l}\text { Tener constancia y saber buscar, investigar, proyectar temáticas; es decir, estar como enterado de } \\
\text { los fenómenos estudiados actualmente en las disciplinas porque eso favorece mucho, el que uno } \\
\text { encuentre bibliografía más actualizada. }\end{array}$ \\
\hline$(24, \mathrm{~F}, \mathrm{~N})$ & Análisis y procesamiento de información. \\
\hline$(30, \mathrm{~F}, \mathrm{~N})$ & $\begin{array}{l}\text { La competencia de alfabetización informacional desde tener claro que necesidad de información tengo, } \\
\text { saber buscar información, evaluarla, procesarla. }\end{array}$ \\
\hline$(28, \mathrm{~F}, \mathrm{~N})$ & Saber hacer una revisión sistemática de toda la literatura de tu área. \\
\hline$(16, \mathrm{~F}, \mathrm{~N})$ & Hay que estar constantemente actualizado revisando las nuevas publicaciones. \\
\hline$(39, \mathrm{M}, \mathrm{E})$ & Habilidades cognitivas mínimas para poder ordenar información. \\
\hline$(34, \mathrm{~F}, \mathrm{~N})$ & Que te guste buscar información y que sepas buscar la mejor sobre tu línea de expertise. \\
\hline$(36, \mathrm{M}, \mathrm{N})$ & Capacidad crítica de análisis de textos. \\
\hline$(48, \mathrm{~F}, \mathrm{E})$ & $\begin{array}{l}\text { Tienen que ir desarrollando habilidades en el sentido de revisar los referentes teóricos de sus áreas } \\
\text { de investigación y una vez que desarrollan los materiales cómo analizar los materiales y evaluarlos. }\end{array}$ \\
\hline
\end{tabular}

El otro código se relaciona con el manejo de software y uso de herramientas tecnológicas en general para las diferentes actividades que se requieren en el proceso de investigación, como lo es el consultar bases de datos, realizar análisis de datos en software especializados o conectarse con el resto del mundo para un fin determinado. En la tabla 2 se muestran algunos de los comentarios de los investigadores entrevistados.

Tabla 2. Respuestas asociadas con el uso de herramientas tecnológicas

\begin{tabular}{|l|l|}
\hline Participante & \multicolumn{1}{c|}{ Respuesta } \\
\hline$(37, \mathrm{M}, \mathrm{N})$ & $\begin{array}{l}\text { Debe conocer de software, sobre todo para el manejo de las bases de datos, para hacer análisis de los } \\
\text { mismos hay que ser muy competentes tecnológicamente hablando. }\end{array}$ \\
\hline$(23, \mathrm{M}, \mathrm{E})$ & Uso de tecnologías para realizar las diversas actividades dentro del proceso de investigación. \\
\hline$(42, \mathrm{M}, \mathrm{N})$ & $\begin{array}{l}\text { También el manejo de tecnología es importante, es increíble pero todavía existen personas que no } \\
\text { están conectadas con esta realidad que es el desarrollo tecnológico. }\end{array}$ \\
\hline
\end{tabular}

\section{Fase. 2 Diseño y validación de la escala}

Con la información de la fase 1 , se generaron las categorías que funcionaron como indicadores y en conjunto con lo encontrado en la literatura, se construyó la escala para medir el nivel de desarrollo de la competencia digital. La escala se conformó por 14 ítems tipo Likert. Con el fin de determinar la fiabilidad, se estimó el estadístico Alfa de Cronbach ( $\alpha$ ) con un valor de .913 y el estadístico omega $(\Omega)$ con un valor de .927. Asimismo, se consideró mantener todos los ítems que presentaron correlaciones con la escala, mayores a .40. De igual forma, para determinar la existencia de correlación entre las variables estudiadas y la necesidad de realizar la técnica factorial se recurrió a la prueba de esfericidad de Bartlett con el índice de medida de adecuación muestral de Kaiser Meyer-Olkim (KMO); dicho índice permite determinar si los reactivos pueden ser interpretados por factor, cuando se obtiene una correlación alta entre las variables con un valor de KMO $>.60$.

Con los puntajes anteriores, se realizó el análisis factorial exploratorio con el fin de conocer el comportamiento de los factores y se indicaron los valores de comunalidad, el valor propio de cada factor, el peso factorial y la 
varianza explicada como se muestra en la tabla 3. En el AFE se usó el método de máxima verosimilitud con rotación varimax. Los coeficientes obtenidos $\left(\mathrm{KMO}=.919\right.$; prueba de esfericidad de Bartlett $\left.X^{2}=2278.77\right)$ determinan la adecuación de los datos para considerar el análisis. En este sentido, se obtuvieron dos factores que determinan el $56.64 \%$ de la varianza total explicada. La correlación entre los factores permite identificar que se está midiendo el mismo constructo.

El análisis factorial confirmatorio de esta escala permitió establecer la bondad de ajuste del modelo para presentar su versión final. Los 14 ítems obtuvieron los puntajes para incluirse en el modelo final (figura 1). Los valores de los indicadores de ajuste obtenidos fueron: $X^{2}=139.20, p=.000 ; \mathrm{CMIN} / \mathrm{DF}=2.0 ; \mathrm{CFI}=.96 ; \mathrm{GFI}=$ $.94 ; \mathrm{NFI}=.94 ; \mathrm{RMSEA}=.05 ; \mathrm{SRMR}=.0389$. En la tabla 3 , se presenta la conformación de la escala final, con las medias y desviaciones estándar, la asimetría, curtosis y comunalidad por ítem.

Tabla 3. Medias, desviaciones estándar, asimetría, curtosis y comunalidad de los ítems que conformaron la escala final de competencias digitales.

\begin{tabular}{|c|c|c|c|c|c|}
\hline İtems & $M$ & $D S$ & Asimetría & Curtosis & Comunalidad \\
\hline $\begin{array}{l}\text { Organizar el cúmulo de información recuperada para } \\
\text { atender una necesidad de investigación específica }\end{array}$ & 3.03 & .789 & -.438 & -.420 & .650 \\
\hline $\begin{array}{l}\text { Evaluar la información recuperada con el fin de } \\
\text { seleccionar aquella pertinente para atender la } \\
\text { necesidad específica }\end{array}$ & 3.08 & .815 & -.599 & -.253 & .661 \\
\hline $\begin{array}{l}\text { Utilizar los sistemas de filtrado que ofrecen las bases } \\
\text { de datos para recuperar la información idónea a mi } \\
\text { necesidad informativa }\end{array}$ & 2.83 & .899 & -.312 & -.735 & .487 \\
\hline Identificar diversas opciones de fuentes de información & 3.10 & .795 & -.547 & -.366 & .628 \\
\hline $\begin{array}{l}\text { Utilizar operadores booleanos (comillas, and, or, not) } \\
\text { para refinar la búsqueda de información }\end{array}$ & 2.64 & .952 & -.106 & -.969 & .325 \\
\hline Saber identificar una necesidad precisa de información & 2.95 & .736 & -.371 & -.135 & .462 \\
\hline $\begin{array}{l}\text { Consultar tesauros como instrumento de control de la } \\
\text { terminología utilizada en la disciplina para realizar las } \\
\text { búsquedas }\end{array}$ & 2.25 & .985 & .392 & -.831 & .358 \\
\hline Buscar información en bases de datos especializadas & 3.24 & .787 & -.834 & 228 & .415 \\
\hline $\begin{array}{l}\text { Analizar los indicadores de revistas (factor de impacto, } \\
\text { citas, indexaciones) }\end{array}$ & 2.62 & .981 & -.065 & -1.02 & .805 \\
\hline $\begin{array}{l}\text { Utilizar índices de factor de impacto para la búsqueda } \\
\text { de información }\end{array}$ & 2.66 & .978 & -.117 & -1.05 & .610 \\
\hline $\begin{array}{l}\text { Seleccionar la revista más adecuada para enviar mi } \\
\text { trabajo }\end{array}$ & 2.56 & 1.00 & -.286 & -1.09 & 630 \\
\hline $\begin{array}{l}\text { Usar herramientas de software para el procesamiento } \\
\text { de datos estadísticos como SPSS, STATA, } \\
\text { STATISTICA, STADIA, R, etc. }\end{array}$ & 2.61 & 1.06 & -.163 & -1.21 & .321 \\
\hline $\begin{array}{l}\text { Conocer las diferentes herramientas tecnológicas para } \\
\text { el desarrollo de un proyecto }\end{array}$ & 2.90 & .823 & -.308 & -.567 & .374 \\
\hline $\begin{array}{l}\text { Utilizar un software de gestor de referencias (EndNote, } \\
\text { Zotero, MENDELEY, BIBLIOSCAPE, } \\
\text { BIBLIOEXPRESS, Scholar's Aid) }\end{array}$ & 2.54 & 1.06 & -.052 & -1.26 & .350 \\
\hline
\end{tabular}

En la tabla 4 se presenta una descripción de los factores que se conformaron con los análisis descritos previamente, la cantidad de reactivos que se incluyeron y los puntajes de fiabilidad aportados por cada factor. Para esta escala, los ítems se ajustaron en dos factores que se orientan a la evaluación de aspectos relacionados a la alfabetización informacional y al uso de herramientas tecnológicas.

Tabla 4. Factores que conforman la escala competencia digital

\begin{tabular}{|l|l|c|c|c|}
\hline Factor & \multicolumn{1}{|c|}{ Descripción } & $\begin{array}{c}\text { Número de } \\
\text { reactivos }\end{array}$ & $\alpha$ & $\Omega$ \\
\hline $\begin{array}{l}\text { Alfabetización } \\
\text { informacional }\end{array}$ & $\begin{array}{l}\text { Habilidades para responder a las necesidades } \\
\text { específicas de información; buscar, discernir y utilizarla } \\
\text { adecuadamente según las labores de investigación; el } \\
\text { manejo de tesauros, índices de factores de impacto, } \\
\text { operadores booleanos; organización y evaluación de } \\
\text { información. }\end{array}$ & 8 & .875 & .878 \\
\hline $\begin{array}{l}\text { Uso de } \\
\text { tecnología }\end{array}$ & $\begin{array}{l}\text { Uso de software, herramientas y gestores para } \\
\text { actividades propias del ámbito científico. }\end{array}$ & 6 & .849 & .847 \\
\hline
\end{tabular}




\section{Descripción de los factores y fiabilidad}

El primer factor se conforma por todo lo que se relaciona con la alfabetización informacional; es decir, con aquellas habilidades que son necesarias para atender una necesidad de información, para generar búsquedas inteligentes, evaluar la cantidad de información de dichas búsquedas, organizarla, seleccionar la idónea y utilizarla adecuadamente en actividades de investigación. Asimismo, conocer el manejo de tesauros, de los índices de factor de impacto y de operadores booleanos, etc. En este factor se agruparon ocho reactivos y en conjunto obtuvieron un Alfa de Cronbach de .875 y un Omega de .878. El segundo factor se conformó por seis ítems que evalúan el manejo operativo de la tecnología que se requiere para realizar actividades de investigación. Es decir, se abordaron indicadores como el uso de software y herramientas para las actividades de análisis de datos, simuladores, etc. Asimismo, para moverse dentro de los gestores de información, base de datos y páginas científicas.

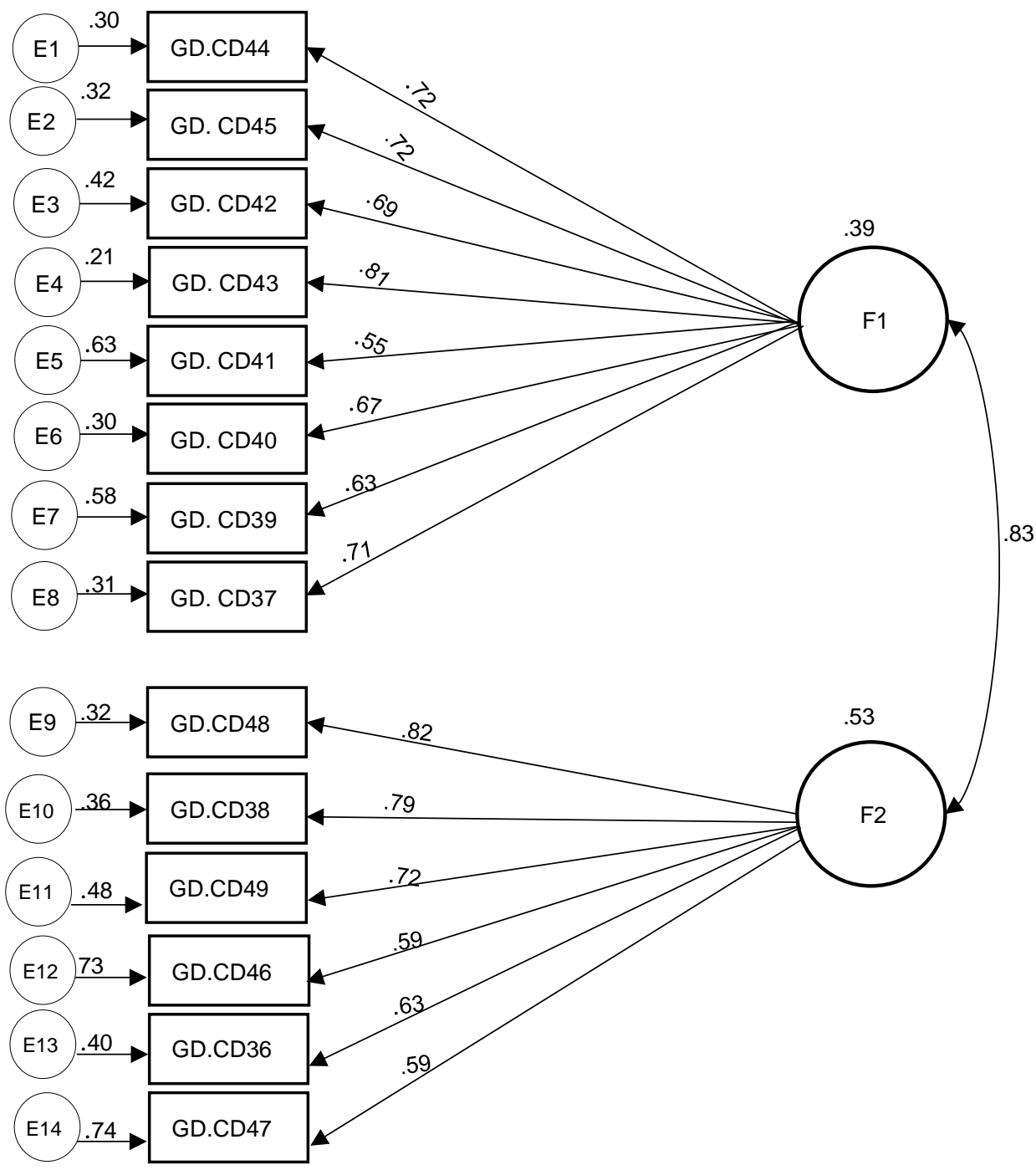

Fig. 1: Resultado del análisis factorial confirmatorio del modelo bidimensional

\section{DISCUSIÓN}

Al revisar la literatura sobre las competencias que se desarrollan en los posgrados se encontraron escalas que miden la competencia digital, pero se enfocaban a un área disciplinar o dejaban fuera indicadores importantes que fueron mencionados en repetidas ocasiones por los investigadores consolidados que se entrevistaron. Este es el caso del estudio de Bolatovna, et al. (2016), quienes trabajaron con la competencia en TIC de estudiantes de maestría. Al igual que el presente estudio, este se interesaba por estudiar las actividades que los estudiantes realizan con el apoyo de tecnología en actividades de investigación científica. Sin embargo, a partir de la información obtenida de las entrevistas a los investigadores, en este estudio se consideró el uso de tecnología solo como una dimensión que compone la competencia digital donde además se requieren habilidades en alfabetización informacional para desempeñarse de forma adecuada como investigador. 
Pinto, et al. (2012) analizaron la competencia informacional de estudiantes de posgrado con una escala de percepción sobre su propia competencia. Es decir, se centraron en la capacidad para saber manejar y comprender la forma sistemática en que la información fluye dentro de los diferentes sistemas; para ello diseñaron el instrumento ALFINVES en el cual se obtuvieron datos correspondientes al saber buscar, encontrar, evaluar y utilizar información con el fin de resolver necesidades. Una de las virtudes que se reportan en este estudio es el ofrecer un diagnóstico general de la evaluación de esta competencia poco abordada en este nivel de estudios. Al igual que el presente trabajo consideramos que la conformación de este tipo de escalas permitirá en un futuro próximo realizar evaluaciones más objetivas como complemento a un reporte de autopercepción, como lo es el evaluar lo que lo que realmente los estudiantes saben hacer.

\section{CONCLUSIONES}

La competencia digital permite el uso eficiente de la tecnología para desarrollarse de forma idónea en actividades relacionadas al trabajo, aprendizaje, ocio y cualquier actividad de participación social. En este sentido, se considera que en la profesión del investigador, para ser un competente digital se requieren sujetos capaces de dominar programas especializados, manejar distintos software para la recopilación y el análisis de datos; interfaces de páginas y aplicaciones diseñados para proyectos y el uso operativo de bases de datos de documentación científica. Asimismo, se debe ser capaz de realizar actividades para manejar la cantidad de información que existe y que permita tener el dominio de conocimiento, detectar la frontera del mismo y proponerse metas en función de ello. Es decir, buscar la información, dónde y cómo recuperarla, gestionarla y evaluarla para estar actualizado en los avances e innovación en el tema y de esta manera estar preparado para abonar al campo de conocimiento con una actualización pertinente.

Es importante aclarar que la propuesta que se presenta es bajo la premisa que dentro de la educación superior los estudios de licenciatura y posgrado requieren de competencias distintas y que los niveles de dominio en los dos grados también son distintos. Asimismo, que dentro de la formación de posgrado existen competencias transversales y específicas de cada una de las áreas de conocimiento. En este sentido, solo se analizaron las que los expertos o investigadores consolidados de la región consideraron como transversales. Es decir, hay habilidades que pertenecen a la competencia digital, que deberían estar presentes en la formación de posgrado y que son determinante para la profesión, pero no resultaron en las entrevistas pues pertenecen a los saberes específicos de cada una de las disciplinas y sería importante generar evidencia de estas con estudios más centrados en ellas.

La escala desarrollada en este trabajo representa una herramienta para evaluar el grado de desarrollo de esta competencia. Este trabajo permite identificar las habilidades que los investigadores consideran conforman una competencia digital que todo investigador con independencia de la disciplina debe dominar; así como la construcción y validación de la escala. Se concluye que la escala diseñada cumple con los criterios de validez y fiabilidad, lo que confirma que posee las propiedades psicométricas para la medición del constructo.

\section{REFERENCIAS}

Asia-Pacific Economic Cooperation, Skills and competencies needed in the research field objectives 2020, Singapore, APEC (2010)

Barthélémy, M.F., y Matthieu, L., The core competencies of PhDs, http://doi.org/10.1080/03075079.2014.968540, Studies in Higher Education, 41 (8), 1355-1370 (2016)

Basantes-Andrade, A., Cabezas-González, M., y Casillas, S., Competencias digitales en la formación de tutores virtuales en la Universidad Técnica del Norte, Ibarra, Ecuador, http://dx.doi.org/10.4067/S0718-50062020000500269, Formación Universitaria, 13(85), 45-56 (2020)

Beltrán, J., Ramírez, M., y García, R.I., Propiedades métricas de un instrumento de autoreporte para medir la competencia informacional de maestros de primaria, http://dx.doi.org/10.12795/pixelbit.2017.i50.10, Pixel Bit, Revista de Medios y Educación, (50), 147-158 (2017)

Blau, I., Shamir-Inbal, T., y Avdiel, O., How does the pedagogical design of a technology-enhanced collaborative academic course promote digital literacies, self-regulation, and perceived learning of students?, https://doi.org/10.1016/j.iheduc.2019.100722, The Internet and Higher Education, 45, 65-78 (2020)

Bolatovna, D., Zhayakovna, A., y otros tres autores, Forming master's degree students' ICT competencies as future researchers and educators: a Kazakhstan case study, http://dx.doi.org/10.15293/2226-3365.1701.03, International Journal of Environmental \& Science Education, 11(18), 11175-11193 (2016)

Carrasco, M., Sánchez, C., y Carro, A., Las competencias digitales en estudiantes del posgrado en educación, https://doi.org/10.22507/rli.v12n2a1, Revista Lasallista de Investigación, 12(2), 10-18 (2015) 
Carretero, S., Vuorikari, R., y Punie, Y., The digital competence framework for citizens, with eight proficiency levels and examples of use, 1a Ed., 10-40, Joint Research Centre, Luxemburgo (2017)

DGTIC., UNAM, Matriz de habilidades digitales, Ciudad de México, México (2014)

Goos, M., y Salomons, A., Measuring teaching quality in higher education: assessing selection bias in course evaluations, https://dx.doi.org/10.1007/s11162-016-9429-8, Research in Higher Education, 58(4), 341-364 (2017)

Hu, L.T., y Bentler, P.M., Cutoff criteria for fit indexes in covariance structure analysis: conventional criteria versus new alternatives, https://doi.org/10.1080/10705519909540118, Structural Equation Modeling: a multidisciplinary journal, 6, 155 (1999)

International Society for Technology in Education, ISTE Standards, Estados Unidos (2015)

Ismail, R., y Mohd, S., Evaluating the research competencies of doctoral students,

https://doi.org/10.1016/j.sbspro.2012.09.271, Procedia Social and Behavioral Sciences, 59, 244-247 (2012)

Joint Information Systems Committee., Building digital capabilities: the six elements defined (2018)

Li, F., y Curdt-Christiansen, X., Teacher feedback in UK higher education: affective and cognitive perceptions of Chinese postgraduate students, https://doi.org/10.1016/j.ijer.2020.101674, International Journal of Educational Research, 104, 225-237 (2020)

López, S., Fortalezas y debilidades de la educación superior en América Latina para la competitividad global, http://dx.doi.org/10.4067/S0718-50062020000500165, Formación Universitaria, 13(5), 165-176 (2020)

Meerah, T.S.M., Osman, K., Zakaria, E., y Ikhsan, Z.H., Developing an instrument to measure research skills, https://doi.org/10.1016/j.sbspro.2012.09.434, Procedia Social and Behavioral Sciences, 60, 630-636 (2012)

Mireles, O., Evaluación de la calidad del posgrado en México, https://doi.org/10.31619/caledu.n29.196, Calidad en la Educación, (29), 242-257 (2008)

OCDE., Higher education in Mexico: labour market relevance and outcomes, higher education, https://doi.org/10.1787/26169177, OCDE, París (2019)

Olehnovica, E., Bolgzda, I., y Kravale, M., Individual potential of doctoral students: structure of research competences and self-assessment, https://doi.org/10.1016/j.sbspro.2015.01.1072, Procedia Social and Behavioral Sciences, 174, 3557-3564 (2014)

Olehnovica, E., Kravale, M., y Bolgzda, I., Doctoral students'competences of using online resources for research purposes in network society, https://doi.org/10.1016/j.sbspro.2013.12.102, Procedia Social and Behavioral Sciences, 106, 896-900 (2013)

Guerrero, G., Tecpan, S., Rojas, S., y Joglar, C., Caracterización del nivel de razonamiento científico en futuros profesores: desafíos para la formación inicial docente, https://dx.doi.org/10.4067/S0718-50062020000500045, Formación Universitaria, 13(85), 45-56 (2020)

Pinto, M., Fernández, A., Sánchez, G., y Meneses, G., Information competence of doctoral students in information science in Spain and Latin America: a self-assessment, https://doi.org/10.1016/j.acalib.2012.08.006, Journal of Academic Librarianship, 39(2), 144-154 (2012)

Traiwichitkhun, D. y Wongwanich, S., Causal model of research competency via scientific literacy of teacher and student, https://doi.org/10.1016/j.sbspro.2014.01.438, Procedia Social and Behavioral Sciences, 116, 1581-1586 (2014)

UNESCO., Enfoques estratégicos sobre las TIC en educación en América Latina y el Caribe, $1^{\text {a }}$ Ed., Chile, OREALC/UNESCO (2013)

Van, E., Jam, A., Van-Dijk, J., y Haan, J., Measuring the levels of 21st-century digital skills among professionals working within the creative industries: a performance-based approach, https://doi.org/10.1016/j.poetic.2020.101434, Poetic, 81, 75-87 (2020) 
